\title{
La integración en las estructuras euroatlánticas. Prioridad de la política exterior de Rumanía ${ }^{1}$
}

\author{
Vasile Dan
}

En el nuevo marco de la globalización e interdependencia la seguridad internacional es ante todo cooperativa e implica estabilidad nacional, democracia y estados de derecho. De esta forma, según el autor, cada Estado miembro de la comunidad debe pretender seguridad de los demás y, al mismo tiempo, suministrarla. En este sentido, Rumania tiene la preparación, la disposición y la capacidad para hacerlo. Dada su vinculación cultural con el viejo continente, la opción sobre su integración en las estructuras europeas y euroatlánticas -Unión Europea, Unión de Europa Occidental y OTAN- es una continuación natural de sus procesos internos y es la manera por la cual se asegura la consolidación y profundización de las reformas internas: sistema democrático, observancia de los derechos humanos, funcionamiento del estado de derecho y economia de mercado.

1. La orientación de la política exterior de un Estado está determinada por la necesidad de promover y defender el interés nacional, que representa, en realidad, la meta fundamental de la politica externa.

La realización de esta meta es posible a través de una correcta evaluación del contexto en que se desarrollan las acciones concretas de política exterior y de los efectos que las mismas acarrean en el plano interno. A su vez, la evolución intema asegura las premisas necesarias

${ }^{1}$ Conferencia dictada en ol Inatituto de Estudios Internacionales de la Universidad de Chile, el dia 26 de noviembre de 1996. 
para el fomento de una política exterior coherente, responsable, transparente y predictible.

La continuación de los procesos de reforma y reconstrucción democrática de la sociedad numana, la afirmación y la consolidación del estado de derecho, el pleno respeto a los derechos humanos, la ampliación del funcionamiento de los principios de la economía de mercado y, no necesariamente en último lugar, la seguridad de la estabilidad interna en un contexto regional caracterizado por agudos: fenómenos de crisis y conflictos abiertos, constituyen componentes de los objetivos de política exterior asignados y fomentados consecuentemente en las acciones específicas de la diplomacia rumana. De este modo, la política exterior ha venido afirmándose como principal instrumento del fomento y la defensa del interés nacional, al lado de, y junto con, la acción desplegada en el plano interno con el mismo fin.

Rumania es parte integrante del espacio de cultura y civilización europeo: fondo cultural latino; cristianismo temprano, fuentes occidentales de formación de la intelectualidad en el siglo. XIX, la política interbélica. Igualmente, ha contribuido de manera propia a su perfil; la expresión de Nicolás Iorga es tan sólo un ejemplo de lo anterior: "Los Estados rumanos.aparecieron.en esta.encrucijada_europea debido a una función económica, política y militar de necesidad europea".

La opción sobre la integración de Rumanía en las estructuras europeas y euroatlánticas es una continuación natural de los: procesos internos anteriormente mencionados. El llevar a la práctica el contenido de las formas políticas y económicas asegura a Rumanía las condiciones necesarias para afirmar su compromiso con los valores generales compartidos por los Estados occidentales que determinaron la aparición y: la evolución de las civilización europea moderna.

Por otro lado, la integración de Rumania en la Unión Europea, la Unión de Europa Occidental y la OTAN es la modalidad por la cual se asegura la evolución de las reformas internas hacia su consolidación y profundización: las condiciones internas que deben cumplirse para el acceso en estas organizaciones prevén la existencia del sistema democrático interno, la observancia de los derechos humanos, el funcionamiento del estado de derecho, la economía de mercado. Al mismo tiempo, la evolución de la sociedad rumana en el marco de estas instituciones se inscribiría naturalmente en la trayectoria perfilada durante casi medio siglo de democracia, estabilidad y bienestar en Europa.

Los últimos años fueron testigos de fenómenos y procesos de fundamental importancia para el porvenir de nuestro continente, especialmente 
en su zona central: el derrumbamiento del comunismo, el fin de la Guerra Fría, la desintegración de la Unión Soviética y el desmantelamiento de Yugoslavia.

Por primera vez en su historia, Rumanía no se avecina más directamente con ninguna gran potencia y las constricciones y los riesgos asociados a semejante vecindad han disminuido considerablemente.

Si en el período de la Guerra Fria Rumanía se encontraba entre cuatro Estados que promovian una ideología común, hoy en dia limita con cinco países con situaciones geoestratégicas $\mathrm{e}$ intereses distintos que gestionan en condiciones y formas específicas sus relaciones externas. Entre estos mismos, Ucrania, República Moldova y la República Federal de Yugoslavia (Serbia y Montenegro), son Estados nuevos e independientes desprendidos no sin condiciones del ex-imperio soviético y la ex-federación yugoslava que están buscando, a su vez, la identidad.

En este contexto, el enfoque pragmático y realista de las perspectivas de promover el interés nacional, lleva a la necesidad de la integración de Rumania a las organizaciones fundamentales, con los valores compartidos y orientados hacia las mismas metas. Al mismo tiempo, la integración europea y euroatlántica ofrece también la posibilidad de afianzar la seguridad y la estabilidad de Rumania, junto con sa inserción en estructuras que comprobaron la viabilidad y la capacidad de adaptación para enfrentar los riesgos y las amenazas:

La altemativa a la integración europea y euroatlántica puede identificarse en dos hipótesis:

- "Deslizamiento" hacia la zona controlada por la Federación Rusa un espacio del tipo "vecindad cercana" a la Comunidad de Estados Independientes - lo que es inaceptable;

- el ingreso en una zona gris, en una postura de neutralidad ilusoria.

Ubicada en las cercanias del frente Sur-Oriental de la Unión Europea y la Alianza del Atlántico Norte, Rumanía es (tras Polonia) el segundo país en tamaño (desde el punto de vista de la superficie, la población, el potencial económico y militar), capaz de asumir y desempeñar activamente un rol importante para guardar y garantizar la seguridad en el centro de Europa y los Balcanes.

En este contexto general, la política exterior rumana persigue aprovechar estas ventajas y su potencial geoestratégico, consolidar su papel estabilizador, así como su concientización en el plano intemacional y ganarse asi la confianza de los factores internacionales de decisión politica y sobre su capacidad de gestionarlos. 
La división Oeste-Este de Europa tuvo como base criterios ideológicos sobre todo. La desaparición del comunismo como sistema en los Estados ex-miembros del Tratado de Varsovia, hizo posible volver a la normalidad geopolitica en el plano de la terminología. En términos geográficos, Rumania se sitúa en Europa Central (las distancias que la separan de las extremidades occidental, nórdica y oriental son iguales). En la dirección Sur, Rumanía está ubicada en la cercanía de la Península Balcánica, manteniendo con los Estados situados en esta región relaciones históricas especiales. Estos elementos justifican la apreciación según la cual Rumanía es un Estado central europeo, con intereses específicos en Europa del Sudeste (Balcanes). En realidad, al comenzar el año 1994, la División para problemas centro-europeos del Departamento de Estado de los Estados Unidos está organizada con dos subdivisiones: Europa Central del Norte y Europa Central del Sur, siendo línea de demarcación las cimas nórdicas de los Montes de los Balcanes.

La preocupación en relación con la ubicación de Rumanía no es académica; su correcta definición permite valorar completamente las ventajas que dimanan del modelo de su percepción para el futuro de la seguridad y la estabilidad del centro del continente con ramificaciones significativas en las zonas adyacentes.

Teniendo en cuenta los recientes acontecimientos de estas zonas (el conflicto de la ex-Yugoslavia y la situación en Transdniester) y el conjunto de las relaciones entre los Estados de las respectivas zonas, destaca fácilmente la importancia que reviste el papel estabilizador y de equilibrio de Rumanía en su región de pertenencia.

2. La integración europea y euroatlántica es un proceso sumamente complejo con implicancias profundas para todas las sociedades involucradas. Los casi 50 años de existencia de los respectivos organismos han llevado a evoluciones estructurales en la organización y el funcionamiento de los sistemas políticos, económicos, sociales, jurídicos . y en el desarrollo de la política exterior y de defensa de los Estados miembros. Ello conlleva a la necesidad de que los Estados que deseen llegar a ser miembros se adapten al conjunto de las reglamentaciones vigentes dentro de las instituciones respectivas. Al mismo tiempo, el ingreso de nuevos miembros supone la necesidad de adaptación de las instituciones respectivas a las condiciones derivadas de la integración de éstos en su actividad.

Rumanía está preparada y dispuesta a integrarse en las instituciones europeas y euroatlánticas en la forma y el ritmo de funcionamiento de 


\section{ESTUDIOS INTERNACIONALES}

las mismas, que regirán en el momento en que se produzca su ingreso a los respectivos organismos. Rumanía cuenta con beneficiarse de las ventajas que emanan de la calidad de miembro de estas instituciones, asumiendo las responsabilidades correspondientes.

Los procesos de ampliación de la Unión Europea y de la OTAN son paralelos, existiendo entre sí ciertos vínculos que, sin condicionarse mutuamente, pueden favorecer a ambos. Su realización deberia conducir entre otras cosas a:

- La acentuación de la estabilidad y la seguridad del continente;

- la ampliación del espacio de desarrollo democrático y bienestar;

- evitar la äparición de nuevas líneas de división del continente;

- $\therefore$ el crecimiento de la capacidad de los Estados miembros de enfrentar las provocaciones y las amenazas a la seguridad y la estabilidad de los mismos y del continente en su conjunto.

Entre las premisas necesarias para alcanzar estos objetivos está la necesidad de un enfoque no discriminatorio de los Estados asociados.(a la Unión Europea) y socios (de la OTAN) en el marco de los procesos de ampliación de las dos organizaciones.

Cabe señalar que en el fomento de la política de integración plena en las estructuras europeas económicas, políticas, de seguridad, culturales, la diplomacia rumana goza del respaldo incondicional del $97 \%$ de la población de Rumanía.

\section{Rumanía y la Unión Europea.}

El diálogo con la Unión Europea y los países miembros ha venido intensificándose a lo largo de los últimos años. Las principales evoluciones han sido marcadas por la entrada en vigencia el 1 de febrero de 1995 del Acuerdo de Asociación; la adopción de la Estrategia Nacional de preparación y entrega de la petición oficial de adhesión del país a la Unión Europea; el envío de la respuesta del gobierno rumano al Cuestionario. de la Comisión Europea sobre la elaboración del visto bueno șobre su candidatura.

Se han creado, al mismo tiempo, mecanismos rumano-comunitarios: el Consejo de Asociación, el Comité de Asociación en el plano gubernamental así como un Comité Interparlamentario de Asociación; existen ya mecanismos nacionales que gestionan el proceso de implementación del Acuerdo de Asociación y de la Estrategia de Adhesión (el Comité Interministerial que tiene al al Primer Ministro de Rumania, el Departa- 
mento de Integración Europea y la Comisión Parlamentaria de Integración). Se han constituido, a la vez, compartimientos especiales de integración a nivel de todas las instituciones centrales.

En este mismo momento la Comisión Europea está estudiando la respuesta del gobiemo rumano al mencionado Cuestionario puesto a su disposición el pasado 25 de julio.

\section{Relaciones con la Unión de Europa Occidental (U.E.o.).}

Rumanía llegó a ser partner asociado a la U.E.O. en mayo de 1994. Hasta' la fecha se destaca la buena cooperación de Rumanía con la U.E.O. en la monitorización de la observancia del régimen de sanciones en el Danubio. Los esfuerzos del país en este campo son reconocidos como una importante contribución a los realizados por la comunidad internacional, con vistas a solucionar por vía política el conflicto de la ex-Yugoslavia.

\section{La colaboración de Rumanía con el Consejo de Europa.}

Rumanía ingresó el 4 de octubre de 1993 como miembro con plenos derechos al Consejo de Europa, lo que significó un importante paso por la vía de su integración en las estructuras políticas europeas. Siguieron la firma y la ratificación de la Convención Europea de los Derechos Humanos y la Convención-marco para la protección de las minorias nacionales en el mismo día del inicio del procedimiento de la firma. $\mathrm{L}$ anterior fue una clara evidencia de la postura de Rumanía a favor de la seguridad y garantía de la observancia de los derechos de las personas pertenecientes a todas las minorías nacionales acorde a los más altos estándares continentales e internacionales.

\section{La colaboración con la Organización de Seguridad y . Cooperación Europeas (O.S.C.E.).}

Se iniciaron y realizaron acciones con vistas a promover, en el contexto euroatlántico, los intereses de seguridad nacional y estabilidad a través de la negociación y la aplicación de medidas capaces de prevenir los conflictos, robustecer la confianza, la seguridad para el control y la limitación de los armamentos, de contribuir al desarrollo, en estrecha 
correlación con las demás estructuras europeas preocupadas en la materia, en las dimensiones económica y humanitaria del proceso de la O.S.C.E.

Rumanía participó activamente en la negociación y la finalización del Pacto de Estabilidad en Europa adoptado por la Conferencia de Paris, de marzo de 1995.

\section{Rumanía y la otan.}

La apertura de la OTAN hacia los Estados de Europa Central persigue ampliar el área de los valores democráticos, los derechos humanos, el estado de derecho, la prosperidad y los principios de la economía de mercado, paralelamente con el proceso de consolidación de la seguridad y la estabilidad continental. Al mismo tiempo, influye el desarrollo de la reforma interna en los Estados postulantes. La apertura de la OTAN debe contribuir al aumento de la seguridad y la estabilidad de los mismos. A su vez, los nuevos miembros están llamados a colaborar efectivamente a la creación del espacio común de seguridad.

Tal como se señalaba anteriormente, Rumanía considera que la realización de las metas perseguidas por el proceso de la apertura de la OTAN, sería posible en las condiciones en que este mismo sea deliberado, gradual y transparente. Un eventual enfoque "en olas" podría afectar.la estabilidad de la zona e induciría a la idea de una discriminación entre los partners que fueron iguales debido a su participación en el Partinership for Peace.

Se puede evitar el surgimiento de nuevas líneas de separación en el continente a través del:

- Mantenimiento del equilibrio geopolítico y de seguridad en Europa Central;

- fomento de un enfoque no competitivo de la apertura para conservar asi los principios definitorios de la Alianza: cohesión, cooperación, acciones conjuntas de seguridad y defensa;

- establecimiento de relaciones de partnership con Rusia y Ucrania; la decisión sobre la firma de una Carta de las relaciones OTAN-Rusia sería el espíritu de este objetivo.

El carácter político de la decisión de aceptación de los candidatos, debe acompañarse del diálogo político (el que está desarrollándose actualmente es preponderantemente militar). A raíz de este diálogo deben establecerse las condiciones de adhesión (un programa individualizado àvanzado de integración), cuyo seguimiento sea examinado periódicamente. 
¿Por qué quiere Rumanía ingresar a la OTAN? ¿Qué es la OTAN hoy en día? ¿Está Rumanía preparada a dar este paso?

$\therefore$ El período subsiguiente a la Guerra Fría creó la oportunidad de la reunificación de Europa en el marco de un espacio común de seguridad. La OTAN comprobó su capacidad de asegurar el desarrollo democrático estable de los Estados miembros y de defender los principios de "la :democracia, la libertad individual y del estado de derecho", tal como se señala en el preámbulo del Tratado de Washington.

Rumanía, que optó firmemente a favor de la plena aplicación de estos principios, considera natural situar como prioridad de su politica exterior el acercamiento a los Estados miembros de la. OTAN con los cuales comparte los mismos valores. Esta opción expresa el componente principal del interés nacional y las encuestas de opinión efectuadas por organismos intermacionales independientes ponen de manifiesto que el 95\% de la población de Rumanía está de acuerdo con la integración del país a la OTAN: No es menos relevante que en el pasado mes de junio de 1996, los partidos parlamentarios adoptaron por unanimidad hacer un llamado dirigido a los parlamentos de los Estados de la OTAN con el fin de que apoyaran las acciones de Rumanía destinadas a su integración en la Alianza.

Al resumir unos cuantos argumentos a favor de esta prioridad rumana de política exterior, me permitiría llamar la atención sobre lo siguiente:

La vida política intema ha venido conociendo una rápida evolución hacia la democracia. En 1991 se adoptó una nueva Constitución que inserta amplias previsiones relacionadas con la aplicación de los principios democráticos, el fomento y la defensa de los derechos humanos. El capítulo dedicado a este tema incluye artículos consagrados a los derechos de las personas pertenecientes a las minorías nacionales, conforme a los cuales las reglamentaciones internacionales en la materia prevalecen sobre las nacionales cuando hayan diferencias entre las mismas.

Las elecciones (locales, parlamentarias y presidenciales) -las más recientes celebradas en noviembre de 1996- se circunscriben al ciclo normal de 4 años de la vida democrática y los debates políticos se centrañ en las modalidades y los medios para profundizar las vías de aplicación de. los principios de la democracia, los derechos humanos, el estado de derecho y la economía de mercado.

La economía de mercado se está ampliando incesantemente: en la agricultura un $95 \%$ de la superficie cultivable pertenece al sector privado. Se está avanzando con el programa de privatización masiva con vistas a acelerar el ritmo de privatización de los objetivos industriales. Hoy en 
dia las sociedades particulares cubren casi un $40 \%$ del comercio exterior de Rumanía y el sector privado participa con casi el $45 \%$ al PIB.

Como Estado central europeo, Rumanía es un verdadero país puente entre el Este-Oeste, el Norte y el Sur. Los elementos específicos de su identidad nacional moldearon la personalidad de los rumanos en por lo menos dos coordenadas principales: el sentimiento de pertenecer. de forma natural a la cultura y la civilización europeas y la extraordinaria disponibilidad de comunicación y apertura hacia otros espacios culturales (Asia Central, Medio Oriente).

Con más de 1:000 kilómetros del curso navegable del Danubio y casi 250 de la orilla occidental al Mar Negro, con el canal MarNegro-Danubio y el gran puerto de Constantza, Rumanía puede ser una verdadera puerta de acceso parecida a lo que Holanda es para Europa.

En el plano militar, entre los socios de la OTAN, Rumanía se encuentra. como uno de los más avanzados en lo que se refiere a la àdaptación de su doctrina militar y el marco legislativo interno está de acuerdo con los requerimientos de la Alianza, inclusive bajo el aspecto del control civil sobre el ejército. La reorganización y la reestructuración del ejército se encuentran en un avanzado estado, acordes a los estándares de la OTAN. La industria militar es cada vez menos dependiente del know-how soviético frente a otros Estados miembros del ex-Tratado de Varsovia. El pasado mes de septiembre, el gobierno decidió crear el Comité Interministerial para la integración de Rumanía en la OTAN en el que están representados los principales ministerios y organismos gubernamentales.

Por su integración rápida en la OTAN, Rumanía pretende contribuir efectivamente a la creación del espacio común de la seguridad, asumiendo, desde luego, todas las obligaciones que le incumben en calidad de miembro de la Alianza.

\section{¿Es Rumanía un factor de estabilidad en la zona?}

Después del cese de la Guerra Fría, el país ha venido probando su - capacidad de ser una zona de estabilidad y seguridad en las condiciones en que había conflictos abiertos en sus cercanías. Para el fomento de una política exterior equilibrada y predictible, Rumanía pudo contribuir directamente a los esfuerzos de la comunidad intemacional para solucionar las crisis. La observancia de las decisiones de la comunidad internacional sobre Irak, Libia y la ex-Yugoslavia, a pesar de las pérdidas internas substanciales causadas por su aplicación, demostró la alineación 
del país a las exigencias de la comunidad internacional y, por consiguiente, la predictibilidad de la política exterior numana.

Dado de que no existen problemas, Rumanía mantiene una postura singular en lo que se refiere a sus relaciones con los Estados del Sudeste de Europa. Es un socio confiable y goza del prestigiố que le brinda sư actitud positiva y equidistante frente a los respectivos Estados.

Las relaciones de buena vecindad y cooperación con los Estados de Europa Central constituyen objetivos constantes de la política exterior rumana. En la actualidad, Rumania ha firmado tratado fundamentales con los seis Estados centro-europeos que intentan integrarse a la OTAN. La finiquitación del Tratado con Hungría representa, en estế sentido, una prueba elocuente del espíritu europeo, en la cual Rumania pretende solucionar aspectos complejos (por su historia y sus implicancias) de las relaciones con los Estados vecinos; al mismo tiempo, ello constituye una contribución concretà à la seguridad y la estabilidad en Europa Central: La firma del Acuerdo de Cooperación entre los Ministerios de Defensa rumano y húngaro, representa, a su vez, un paso concreto hacia el afianzamiento de la confianza mutua e, implícitamente, de la estabilidad de la región.

En sus relaciones con la República de Moldova, Rumania parte del reconocimiento de la soberanía la independencia y la integridad territorial de ese Estado y actúa a favor de su consolidación: Entre los dos Estados existen relaciones especiales, orientadas hacia la integración económica y la consolidación del acervo espiritual y cultural comün.

Ucrania es, en la actualidad, el más grande vecino de Rumanía. En los vínculos bilaterales, Rumania está vivamente interesada én la consolidación de la estatalidad de ese país y en su afirmación comón pạticipante importante en la arquitectura de seguridad europea. La negociación del Tratado fundamental con Ucrania se está finiquitando.

Las relaciones con Bulgaria, reglamentadas por el Tratado fundamental en vigencia desde 1993, revisten un carácter constructivo tradicional Este acuerdo brinda un notable potencial de cooperación especialmente en el ámbito de la infraestructura.

Frente a los Estados de la ex-Yugoslavia, Rumanía tuvo una actitud equilibrada y equidistante, lo que le aseguró la posibilidad de mantener un diálogo político intenso durante la crisis. Su directa implicancia en el proceso de paz y reconstrucción representa una expresión concreta de la contribución a la normalización de la situación en la región. En el plano jurídico, Rumania firmó tratados fundamentales con la República Federativa de Yugoslavia y Croacia, mientras que los acuerdos con Eslovenia y Macedonia están en la fase final. 
Como se decía antes, por vez primera en su historia modema, Rumanía no tiene más fronteras comunes con Rusia. Claro está que el peso y la importancia de Rusia determinan el interés de Rumanía para enfocar las relaciones rumano-rusas desde la perspectiva de la importancia que tiene el apoyo de la evolución hacia la democracia y la economía de mercado en ese país, como garantía potencial de la estabilidad en la región.

Rumanía aprecia, al mismo tiempo, que en el proceso de estabilidad y seguridad en Europa Central un rol importante le incumbe a la cooperación subregional y actúa como tal siendo miembro con plenos derechós en la Organización de Cooperación en la zona del Mar Negro, la Iniciativa y la Asociación Centroeuropeas del Libre Comercio (CEFTA).

El enfoque equilibrado, realista y global de las relaciones internacionales actualés y de su dinámica conllevá al establecimiento de prioridades de integración de Rumanía en las estructuras europeas y euroatlánticas. Este proceso se despliega en dos niveles: interno -profundizar y acelerar la reforma-y externo -desarrollar las relaciones con las respectivas instituciones y con los Estados miembros en el plano bilateral, para robustecer y favorecer, a su vez, los lazos de cooperación, partnership y amistad con todos los Estados del mundo-. Finalmente, la implicancia cada vez más sustantiva de Rumanía en el conjunto de las relaciones globales es una condición fundamental para promover el interés nacional en un mundo cada vez más interdependiente.

La reciente declaración del mandatario norteamericano hecha en Detroit poco antes de su reelección, ofrece un horizonte de tiempo más realista y una perspectiva clara, abierta a todas las nuevas democracias interesadas y preparadas a ingresar a la alianza noratlántica.

Rumanía cuenta con la ayuda de los Estados Unidos de Norteamérica y de los demás Estados miembros de la OTAN para que se cumpla el objetivo declarado de integrar las filas de los primeros países con los cuales ha de comenzar el proceso de ampliación de la Alianza. Rumanía hará todo lo que de ella dependa para seguir actuando consecuentemente, con firmeza y responsabilidad para alcanzar esta meta.

La seguridad internacional tiene más que nunca un carácter indivisible, es cooperativa e implica estabilidad nacional, democracia y estado de derecho. Cada Estado miembro de la comunidad internacional debe pretender seguridad de los demás y tiene que brindar o suministrar, a su vez, seguridad.

Rumanía está preparada, dispuesta y capacitada para realizarlo. 